\title{
Spinal Cord Compression Due to a Catheter Tip Granuloma of an Intrathecal Morphine Pump
}

Gaurav Jain, $\mathrm{MD}^{1 *}$; Nashaat N. Rizk, MD²

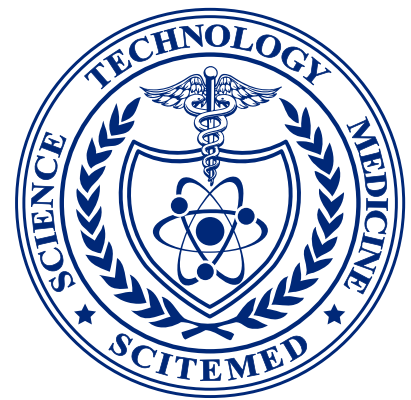

${ }^{1}$ Department of Anesthesiology, Division of Pain Management, Berkshire Medical Center, Pittsfield, MA, USA

2Department of Anesthesiology, West Virginia University, Morgantown, WV, USA

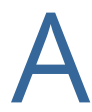
59-year-old woman had an intrathecal morphine pump therapy for the last 20 years, due to chronic lumbar back pain and L4,5 post-laminectomy syndrome, and was presented with 6 days onset of lower thoracic back pain accompanied with feelings of heaviness and tingling below mid-thigh level bilaterally. There was no change in the drug concentration $(20 \mathrm{mg} / \mathrm{ml})$ within the last four years. She denied having fever, headache, confusion, saddle anesthesia, bladder or bowel incontinence and history of cancer. Upon examination she had decreased pin-point sensation, and absent vibration sense below mid-thigh level bilaterally with intact motor exam.

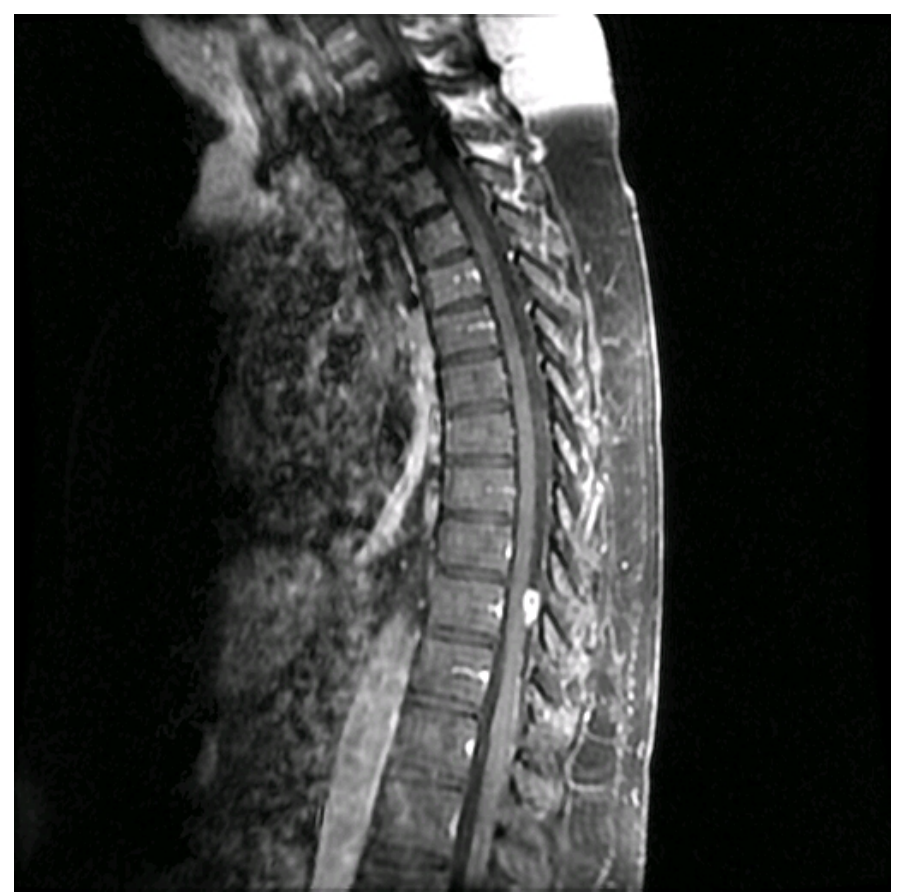

Figure 1. Gadolinium contrast enhanced mid-line sagittal view of thoracic spine MRI. intraspinal extramedullary ring enhancing lesion at T10 level abutting the spinal cord posterior-laterally on the right side. It measured $14.8 \mathrm{~mm}$ superior to inferior and $7.7 \mathrm{~mm}$ anterior to posterior abutting the spinal cord posterior-laterally on the right side with no signs of inflammation. MRI, magnetic resonance imaging.
The gadolinium enhanced magnetic resonance imaging (MRI) of thoracic spine showed an intraspinal extramedullary ring enhancing lesion at T10 level abutting the spinal cord posterior-laterally on the right side (Figures 1-6). MRI of the head, and the cervical and lumbar spines were unremarkable. Cerebrospinal fluid analysis was normal and cultures were negative for infection. She was diagnosed with catheter tip granuloma [1,2] and the intrathecal morphine was stopped. Pump was operated with normal saline, and serial thoracic MRI was obtained every week until symptoms were resolved after 2 weeks; and granuloma was resolved after 3 weeks.

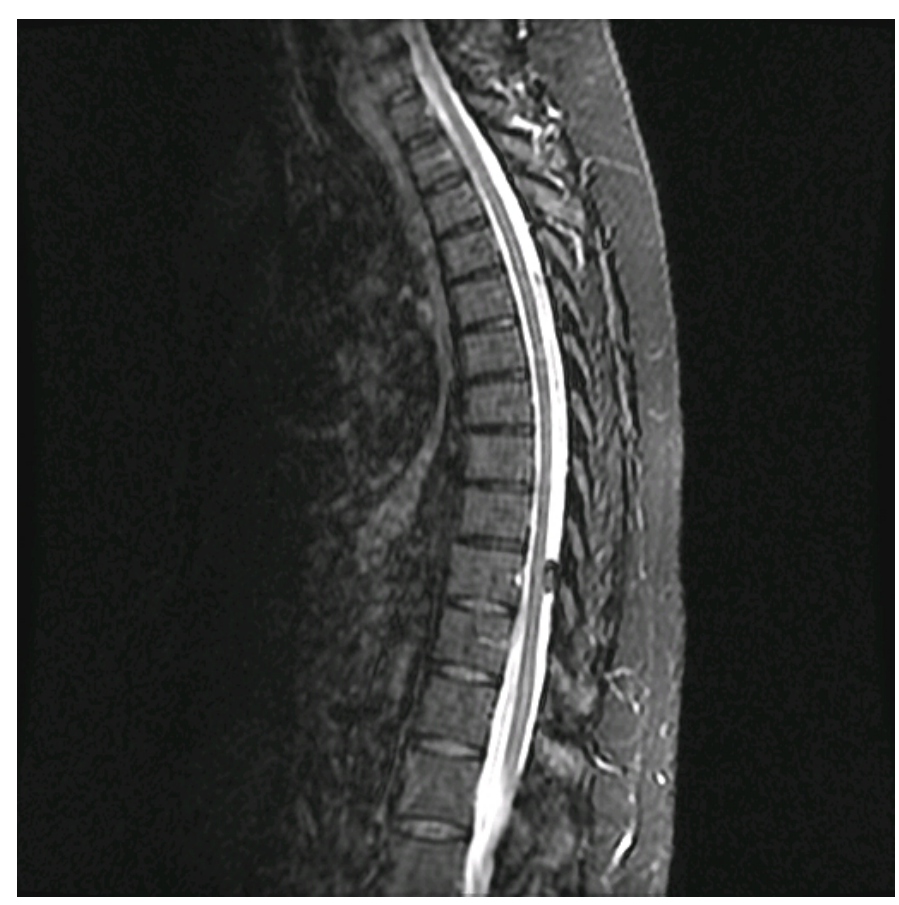

Figure 2. STIR thoracic MRI mid-line sagittal view with no signs of inflamation. MRI, magnetic resonance imaging; STIR, short inversion time inversion recovery. 


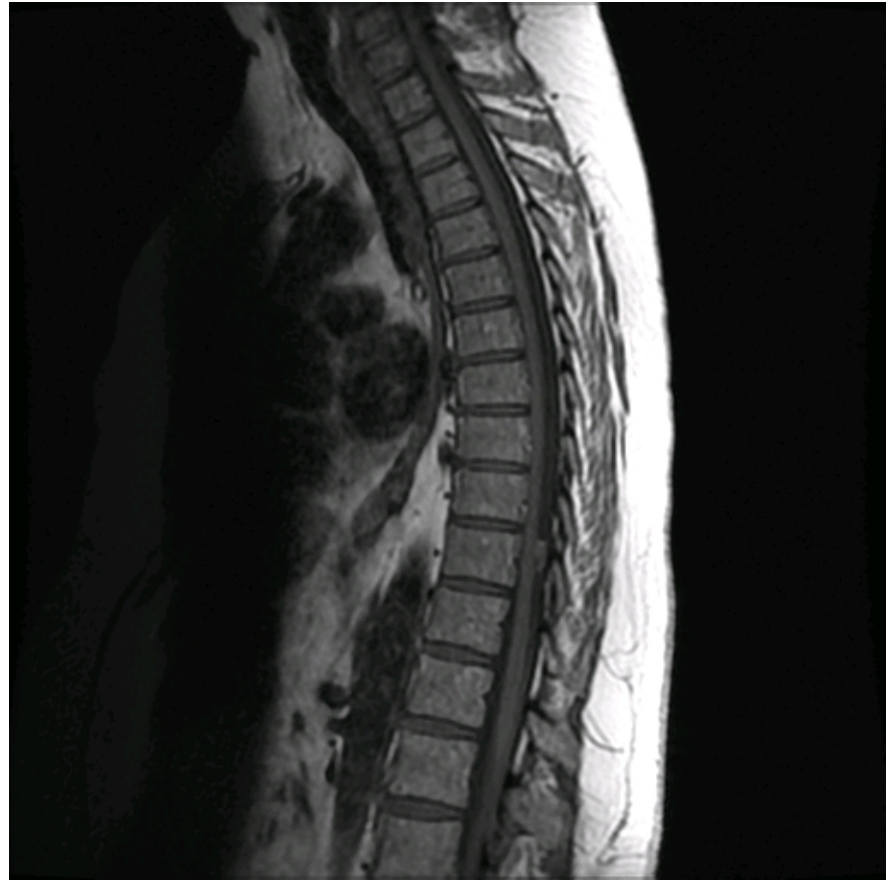

Figure 3. T1-weighted thoracic MRI mid-line sagittal view. MRI, magnetic resonance imaging.

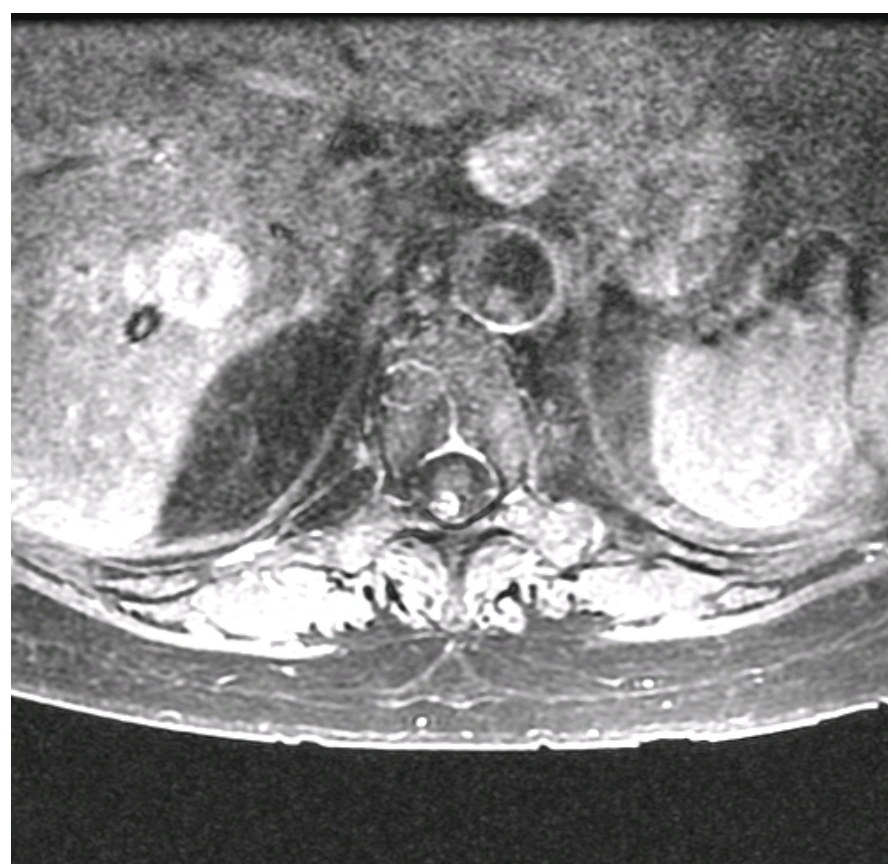

Figure 5. Gadolinium contrast enhanced transverse view of thoracic spine MRI at upper T10 level. MRI, magnetic resonance imaging.

\section{Article Information}

*Correspondence: Gaurav Jain, MD

Division of Pain Medicine, Department of Anesthesiology, Berkshire Medical Center, Pittsfield, MA, USA. Email: drgauravjain@gmail.com

Received: Jul. 26, 2017; Accepted: Oct. 27, 2017; Published: Jun. 20, 2018

DOI: $10.24983 /$ scitemed.nnr.2018.00066

Copyright $(2018$ The Author(s). This is an open-access article distributed under the terms of the Creative Commons Attribution 4.0 International License (CC-BY).

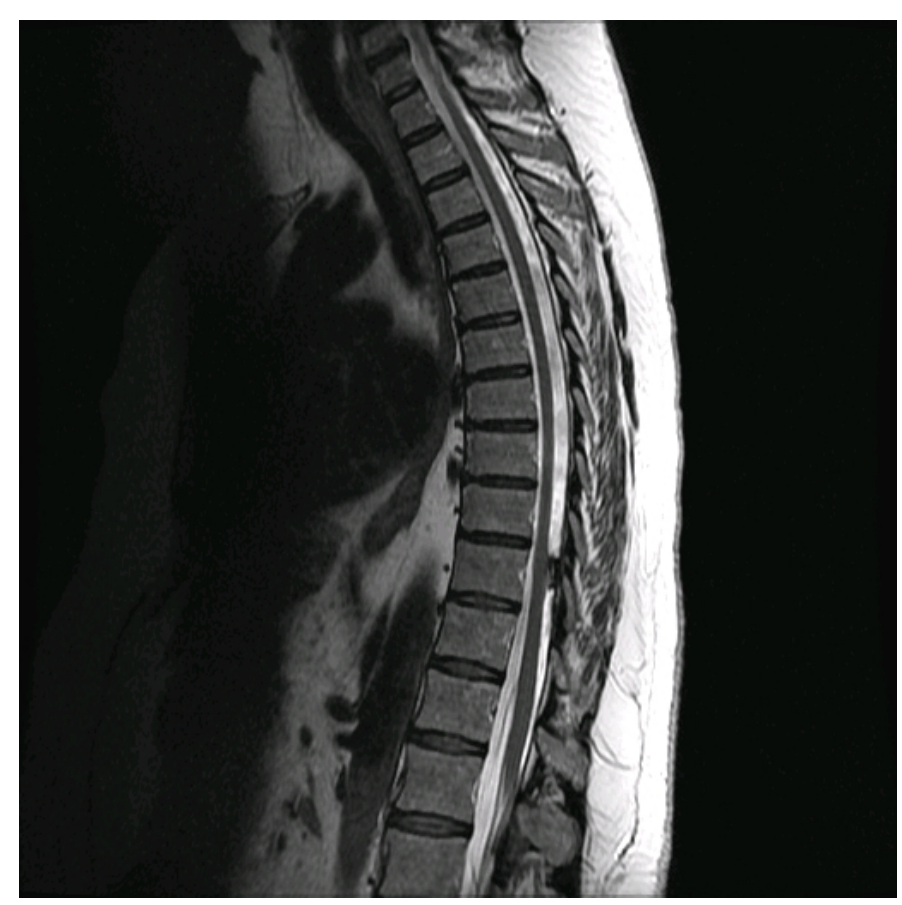

Figure 4. T2-weighted thoracic MRI mid-line sagittal view. MRI, magnetic resonance imaging.

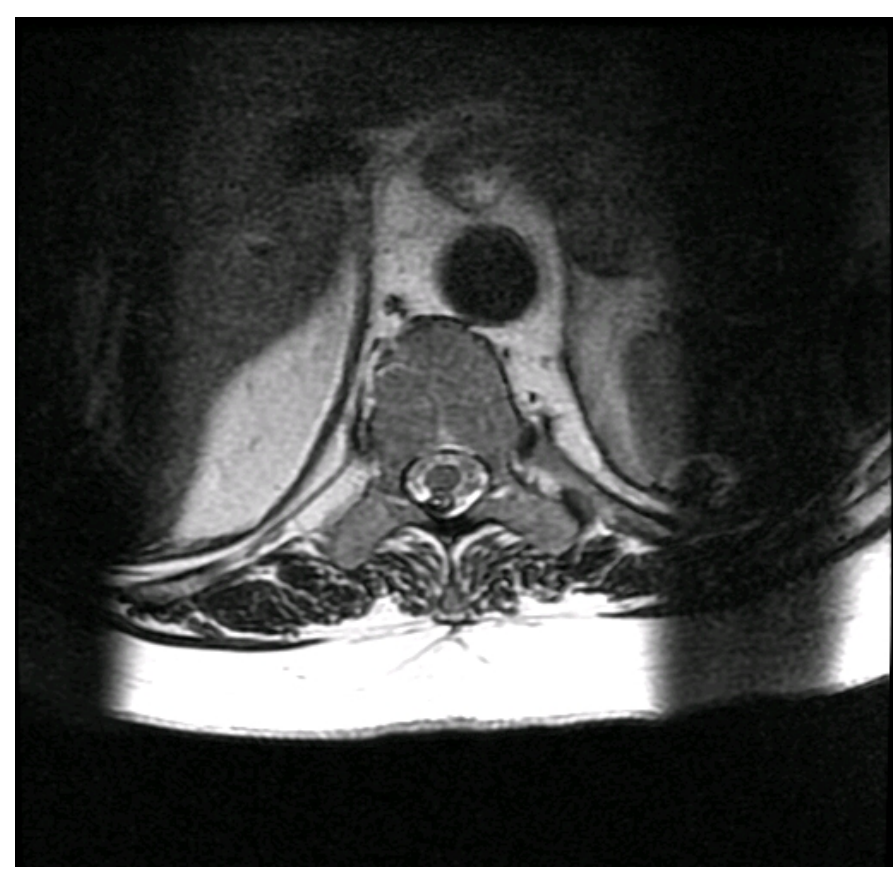

Figure 6. T2-weighted transverse view of thoracic spine MRI at upper T10 level. MRI, magnetic resonance imaging.

Funding: None

Conflict of Interest: None

\section{References:}

1. Coffey R, Burchiel K. Inflammatory mass lesions associated with intrathecal drug infusion catheters: report and observations on 41 patients. Neurosurgery 2002;50:78-86; discussion 86-87.

2. Deer TR. A prospective analysis of intrathecal granuloma in chronic pain ppatients: a review of the literature and report of a surveillance study. Pain Physician 2004;7:225-228. 